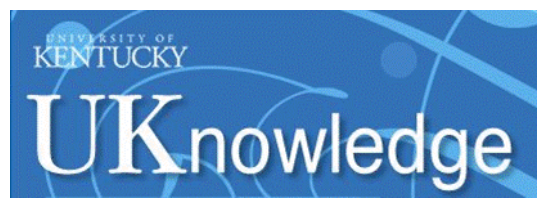

University of Kentucky

UKnowledge

7-20-2001

\title{
Measurement and Interpretation of Deuterium-Line Emission in the Orion Nebula
}

C. R. O'Dell

Vanderbilt University

Gary J. Ferland

University of Kentucky, gary@uky.edu

W. J. Henney

UNAM Campus Morelia, Mexico

Follow this and additional works at: https://uknowledge.uky.edu/physastron_facpub

Part of the Astrophysics and Astronomy Commons, and the Physics Commons

Right click to open a feedback form in a new tab to let us know how this document benefits you.

\section{Repository Citation}

O'Dell, C. R.; Ferland, Gary J.; and Henney, W. J., "Measurement and Interpretation of Deuterium-Line Emission in the Orion Nebula" (2001). Physics and Astronomy Faculty Publications. 106.

https://uknowledge.uky.edu/physastron_facpub/106

This Article is brought to you for free and open access by the Physics and Astronomy at UKnowledge. It has been accepted for inclusion in Physics and Astronomy Faculty Publications by an authorized administrator of UKnowledge. For more information, please contact UKnowledge@lsv.uky.edu. 


\section{Measurement and Interpretation of Deuterium-Line Emission in the Orion Nebula Digital Object Identifier (DOI) https://dx.doi.org/10.1086/321562 \\ Notes/Citation Information \\ Published in The Astrophysical Journal, v. 556, no. 1, p. 203-214. \\ (C) 2001. The American Astronomical Society. All rights reserved. Printed in the U.S.A. \\ The copyright holder has granted permission for posting the article here.}


The Astrophysical JournaL, 556:203-214, 2001 July 20

(C) 2001. The American Astronomical Society. All rights reserved. Printed in U.S.A.

\title{
MEASUREMENT AND INTERPRETATION OF DEUTERIUM-LINE EMISSION IN THE ORION NEBULA ${ }^{1,2,3}$
}

\author{
C. R. O’DELL \\ Department of Physics and Astronomy, Vanderbilt University, Box 1807-B, Nashville, TN 37235; cr.odell@vanderbilt.edu \\ G. J. FERLAND \\ Department of Physics and Astronomy, 177 CP Building, University of Kentucky, Lexington, KY 40506-0055 \\ AND \\ W. J. HENNEY \\ Instituto de Astronomía, UNAM Campus Morelia, Apartado Postal 3-72 (Xangari), 58089 Morelia, Michoacán, México \\ Received 2001 February 7; accepted 2001 March 29
}

\begin{abstract}
We present new observations of the deuterium and hydrogen Balmer lines in the Orion Nebula. There is a real variation in the deuterium-to-hydrogen line ratios across the nebula, being greatest in the emission from the largest proplyd (Orion 244-440). We also present the results of a detailed model for the emission of these lines, the hydrogen lines being the result of photoionization and recombination while the deuterium lines are produced by fluorescent excitation of the upper energy states by the far-UV radiation from $\theta^{1}$ Ori C. Comparison of the observations and predictions of the line intensities shows good agreement, both in the strength of the reference lines at $\mathrm{H} \beta$ and also in the differences of the Balmer decrement for the two atoms. The fact that both the deuterium and hydrogen emissions arise from mechanisms that count the near-ultraviolet (deuterium) and photoionizing ultraviolet (hydrogen) photons from the dominant star means that there is little prospect of similar observations being useful for determination of $\mathrm{D} / \mathrm{H}$ abundances in $\mathrm{H}$ II regions.
\end{abstract}

Subject headings: ISM: abundances — ISM: general — ISM: individual (Orion Nebula)

\section{INTRODUCTION}

The abundance of deuterium is an important but difficult guide to the chemical evolution of the universe: "important" because it is believed to have been formed in the conditions of the Big Bang and has been monotonically destroyed as material is cycled through stars, and "difficult" because the abundance is so low and its lines so difficult to observe that accurate determinations have been rare (see the excellent summary by Bertoldi et al. 1999 of the current results). Early efforts at detection in $\mathrm{H}$ iI regions were reported to be unsuccessful, but Hébrard et al. (2000a, hereafter HA) made a convincing measurement of deuterium in emission in the shoulder of $\mathrm{H} \alpha$ and $\mathrm{H} \beta$ lines in the Orion Nebula, a discovery quickly followed by detections in M8, M16, M20, and DEM S 103 in the Small Magellanic Cloud (Hébrard et al. 2000b, hereafter HB). The key to the identifications was recognition that the deuterium Balmer emission lines would not occur at the isotopic shift of -82 $\mathrm{km} \mathrm{s}^{-1}$ with respect to the hydrogen lines if the deuterium arose from a region with a different radial velocity than that of hydrogen. Since the Orion Nebula is a blister of ionized

\footnotetext{
${ }^{1}$ Based in part on observations with the NASA/ESA Hubble Space Telescope, obtained at the Space Telescope Science Institute, which is operated by the Association of Universities for Research in Astronomy, Inc., under NASA contract NAS5-26555.

${ }^{2}$ Based in part on observations obtained at the Kitt Peak National Observatory, which is operated by the Association of Universities for Research in Astronomy, Inc., under a cooperative agreement with the National Science Foundation.

${ }^{3}$ Some of the data presented herein were obtained at the W. M. Keck Observatory, which is operated as a scientific partnership among the California Institute of Technology, the University of California, and the National Aeronautics and Space Administration. The Observatory was made possible by the generous financial support of the W. M. Keck Foundation.
}

gas on the facing side of the molecular cloud OMC-1, it is characterized by emission starting from $28 \mathrm{~km} \mathrm{~s}^{-1}$ for OMC-1 to smaller values for states of higher ionization (O’Dell 2001), hydrogen recombination lines being found at about $18 \mathrm{~km} \mathrm{~s}^{-1} .4$ By noting that the weak lines on the blue shoulder of the Balmer lines were narrower than the thermal width for the ionized gas and fell at a wavelength corresponding to $-82 \mathrm{~km} \mathrm{~s}^{-1}$ with respect to OMC-1, HA argued that the deuterium emission arises from fluorescence with stellar continuum photons below $13.6 \mathrm{eV}$ in the photon dominated region (PDR) lying just beyond Orion's main ionization front (IF). Since the surface brightness in an ionized blister is directly proportional to the incident photoionizing flux of photons (Baldwin et al. 1991, hereafter BFM) and the surface brightness in a continuum-pumped deuterium line is dependent on the flux of those lower energy photons, it is not surprising that the deuterium lines were detected at levels much in excess of those expected from the abundance ratios measured elsewhere.

It is this surprising strength and displacement with respect to the hydrogen lines that has caused previous investigators to measure the deuterium lines but fail in their identification. The PEPSIOS study by Traub, Carleton, \& Hegyi (1974) measured the line at up to 0.003 times the intensity of $\mathrm{H} \alpha$ at a relative velocity of about $-60 \mathrm{~km} \mathrm{~s}^{-1}$. In a recent study of the $\mathrm{Br} \gamma$ line, Oudmaijer et al. (1997) detected what we argue to be the deuterium component at $-70 \mathrm{~km} \mathrm{~s}^{-1}$ relative velocity but dismissed its reality because they could not see a corresponding component in their $\mathrm{H} \beta$-line profile. We will show in this paper that the

\footnotetext{
${ }^{4}$ All radial velocities in this paper will be heliocentric. To obtain radial velocities with respect to the local standard of rest, subtract $18.1 \mathrm{~km} \mathrm{~s}^{-1}$.
} 


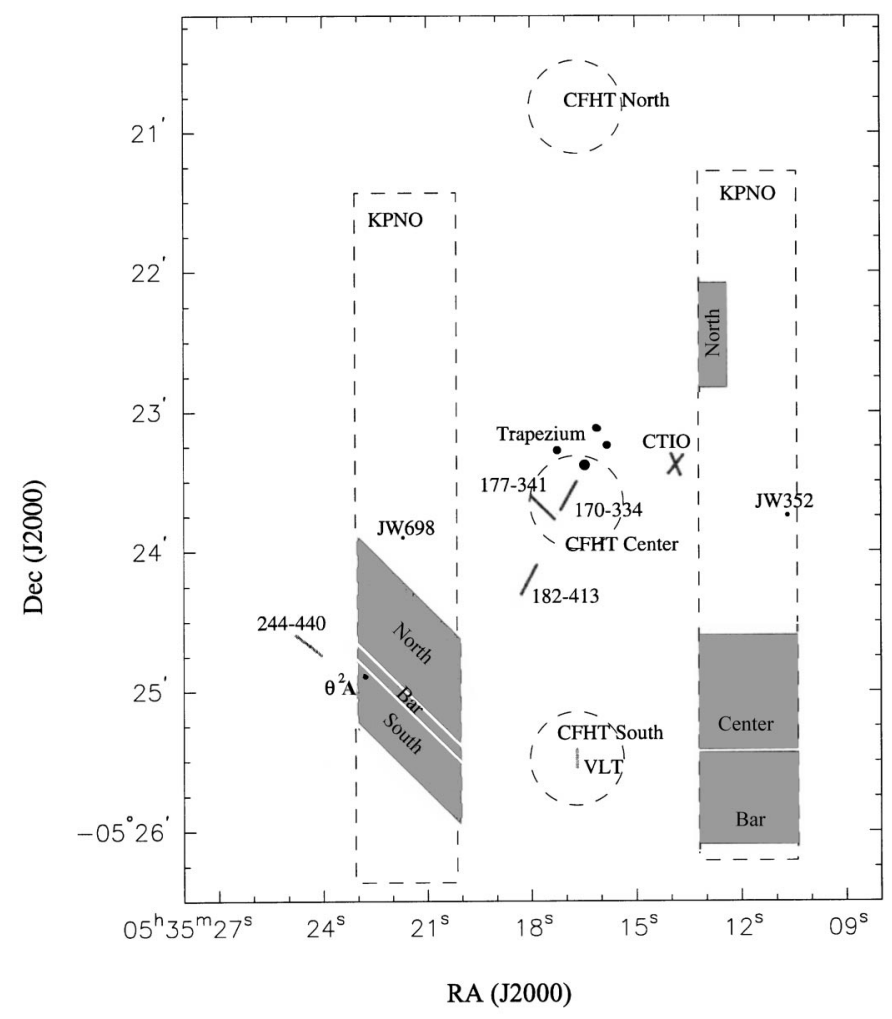

FIG. 1.-Positions of slit apertures used in all the different observational data sets represented in Table 1 . Larger filled circles show the $O B$ stars of the Trapezium $\left(\theta^{1}\right.$ Ori A, B, C, D) and $\theta^{2}$ Ori A for ease of orientation. Smaller filled circles show the guide stars JW352 and JW698 used for the KPNO spectra. The total areas covered by these spectra are indicated by the dashed rectangular boxes, whereas the filled gray areas indicate those regions for which deuterium characteristics were extracted. The four areas covered by the Keck spectra are indicated by gray $14^{\prime \prime}$ slits, each labeled with the coordinate name of the proplyd on which it was centered. For the CTIO study, the $10^{\prime \prime}$-slit segment is indicated at the position angles used for $\mathrm{H} \alpha\left(142^{\circ}\right)$ and other lines $\left(203^{\circ}\right)$. Also shown is the slit used in the Very Large Telescope study of HB and the three regions (dashed circles) covered by a rotating slit in the Canada-France-Hawaii Telescope observations of HA.

relative intensities of the deuterium and hydrogen lines are far from constant, selectively becoming relatively stronger for lines arising from higher quantum states $n$. The radial velocity of the PDR is $10 \mathrm{~km} \mathrm{~s}^{-1}$ redshifted with respect to the hydrogen recombination lines; therefore, the earliest studies were detecting the deuterium lines but failed to identify them because of the unexpected velocities.

\section{THE OBSERVATIONS}

Three sets of high-resolution CCD spectra of the Orion Nebula were used in this study, these providing the wide dynamic range necessary for attacking the problem of accurate measurement of weak deuterium emission-lines on the shoulders of the hydrogen lines, which are at least $10^{3}$ times stronger. The first data set is spectra obtained with the Keck 1 HIRES spectrograph (Vogt et al. 1994) as a part of a study of mass loss from proplyds through photoevaporation. The details of the observations are described in Henney \& O'Dell (1999). Suffice it to say that these were $14^{\prime \prime}$-long-slit spectra centered on individual bright proplyds and had instrumental FWHM of $6.2 \mathrm{~km} \mathrm{~s}^{-1}$. These spectra covered both $\mathrm{H} \alpha$ and $\mathrm{H} \beta$. The second data set was obtained with the echelle spectrograph mounted on the $4 \mathrm{~m}$ telescope at Kitt Peak National Observatory (KPNO). In this case the instrumental FWHM is $8.4 \mathrm{~km} \mathrm{~s}^{-1}$. The entrance slit was not constrained by a decker but rather by the vignetting of the optical train, with a 336"-long sample taken. A 0"8-wide, north-south-orientation slit was stepped across two regions of the nebula, the first centered at $\delta=-5^{\circ} 23^{\prime} 54^{\prime \prime}\left(\right.$ E2000) and covering R.A. $=5^{\mathrm{h}} 35^{\mathrm{m}} 20^{\mathrm{s}} \cdot 18$ $5^{\mathrm{h}} 35^{\mathrm{m}} 23^{\mathrm{s}} .19$ and the second centered at $\delta=-5^{\circ} 23^{\prime} 45^{\prime \prime}$ and covering R.A. $=5^{\mathrm{h}} 35^{\mathrm{m}} 10^{\mathrm{s}} \cdot 40-5^{\mathrm{h}} 35^{\mathrm{m}} 13 \mathrm{~s} .21$. With only a few exceptions, steps of $2^{\prime \prime}$ in right ascension were used. These spectra were obtained as part of the Ph.D. thesis work of Takao Doi, who is deriving velocity maps of the Orion Nebula at $10 \mathrm{~km} \mathrm{~s}^{-1}$ resolution in multiple ions, and the easternmost of the samples covered the "bright bar" feature of the Nebula, which is argued to be a section of the main ionization front that is viewed nearly edge-on. These provided spectra of the $\mathrm{H} \alpha$ line and also lines of much higher ([O III]) and lower ([S II $]$ ) ionization, which were useful for checking that the putative deuterium lines were not actually highly blueshifted shocked material (there was no evidence for this). The third data set was spectra made by Jack Baldwin with the $4 \mathrm{~m}$ echelle spectrograph at the Cerro Tololo Inter-American Observatory (CTIO). These spectra were obtained as part of a program to accurately measure and identify weak emission lines in Orion (Baldwin et al. 2000) and are with a $10^{\prime \prime}$-long-slit sample centered at $05^{\mathrm{h}} 35^{\mathrm{m}} 13.9^{\mathrm{s}},-05^{\circ} 23^{\prime} 23^{\prime \prime}$ and at position angles of $142^{\circ}$ and $203^{\circ}$, which aligned the slit along the direction of the atmospheric dispersion, hence increasing the photometric accuracy. The spectrograph and resolution are essentially identical to the Kitt Peak observations, but the longest observations of $3 \times 1000 \mathrm{~s}$ exposures had much greater signal than the $2 \times 150 \mathrm{~s}$ KPNO exposures. However, the total signals were comparable because of the multiple-slit pointings at KPNO. All of these spectra were processed using the IRAF $^{5}$ data analysis package. The particular advantage of the CTIO data set was that the spectral coverage extended to $3510 \AA$, thus allowing measurement of the higher Balmer lines. The locations of the various slit settings are shown in Figure 1.

\section{DERIVATION OF DEUTERIUM- AND HYDROGEN-LINE CHARACTERISTICS}

The appearance of the deuterium lines as weak features on the blue wing of the hydrogen Balmer lines has been demonstrated in HA and HB and is illustrated in Figure 2, which shows an example spectrum from our Keck data set. Our data are of very similar appearance to those in HA and $\mathrm{HB}$, although the signal-to-noise ratio $(\mathrm{S} / \mathrm{N})$ is usually higher, and in some cases we go to higher members of the Balmer series. Grating ghosts are present in the CTIO spectra but are sufficiently widely spaced that they are not problems except for the highest Balmer lines.

In this section, we first outline the general methods used to extract the deuterium components and then present in turn the results from each of our three data sets, before

\footnotetext{
${ }^{5}$ IRAF is distributed by the National Optical Astronomy Observatories, which are operated by the Association of Universities for Research in Astronomy, Inc., under cooperative agreement with the National Science foundation.
} 


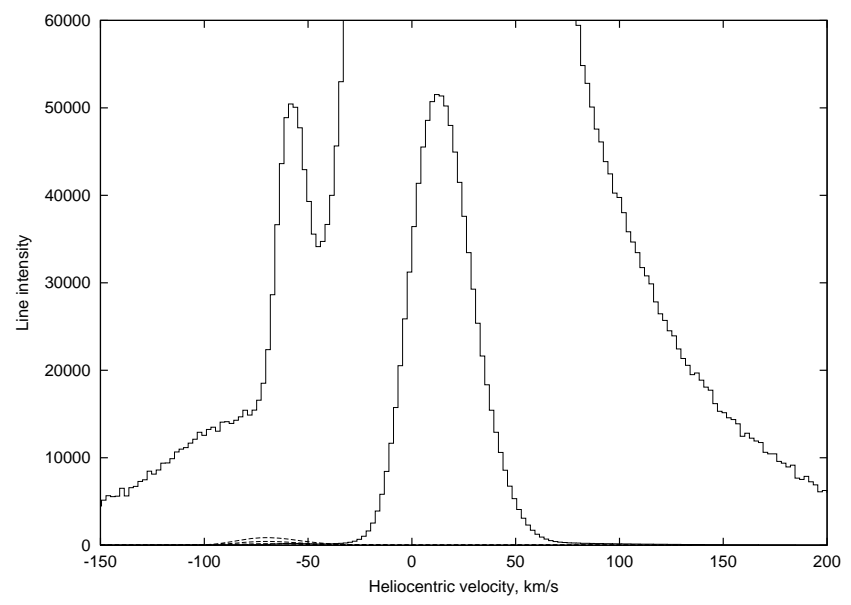

FIG. 2.-Observed $\mathrm{H} \alpha$-line profile of the giant proplyd $244-440$ shown at $1 \times$ and $250 \times$ magnification. The magnified profile clearly shows the fluorescent $\mathrm{D} \alpha$ component at $-60 \mathrm{~km} \mathrm{~s}^{-1}$ (velocity scale is relative to $\mathrm{H} \alpha$; add $81.6 \mathrm{~km} \mathrm{~s}^{-1}$ for velocity relative to $\mathrm{D} \alpha$ ). Also shown (dashed lines) are $250 \times$ magnifications of the expected recombination $\mathrm{D} \alpha$ component, assuming relative abundances $\mathrm{D} / \mathrm{H}=(2,5,10) \times 10^{-5}$.

finally discussing the consistency between our results and those of HA and HB.

\subsection{General Methodology}

We employed three different methods for extracting the deuterium lines from the hydrogen wings. In the first method, a low-order polynomial was fitted to the wing in a small velocity range centered on the deuterium component, and this was subtracted to give the deuterium-line profile. This method is applied to the spectrum at each spatial position along the slit and works best where the $\mathrm{S} / \mathrm{N}$ is high and the deuterium component is well separated from the Gaussian core of the hydrogen line. Best results are obtained for the Keck HIRES spectra, and an example position-velocity image of an extracted $\mathrm{D} \alpha$ line from Keck spectra of the giant proplyd 244-440 is shown in Figure 3.

For spectra where the $\mathrm{S} / \mathrm{N}$ was lower (in particular, the KPNO data), it was found necessary to use a much wider velocity range in order to fit the hydrogen wing, which cannot be well represented by a low-order polynomial. Hence, in this second method, we fitted a sum of a polynomial, a Lorentzian, and a Gaussian to the hydrogen wing in order to isolate the deuterium profile.

A third, manual, graphical method was also employed as a sanity check on the two automated techniques. In this method, a series of spectra from a spatial region were summed and plotted as a linear or semilogarithmic profile. A smooth curve was then hand-drawn through the blue hydrogen wing and subtracted to give the deuterium profile (after taking antilogs in the case of semilog plots). In the case of the CTIO spectra, this technique was helped by folding the red wing over onto the blue wing, but this was not possible in the case of the other data sets due to asymmetries in the instrumental profile.

The results of applying these techniques are summarized in Table 1 for the three different data sets presented in this paper (further details on individual data sets are given below). Also shown, for comparison, are the results from the published studies of HA and HB. The spatial locations of

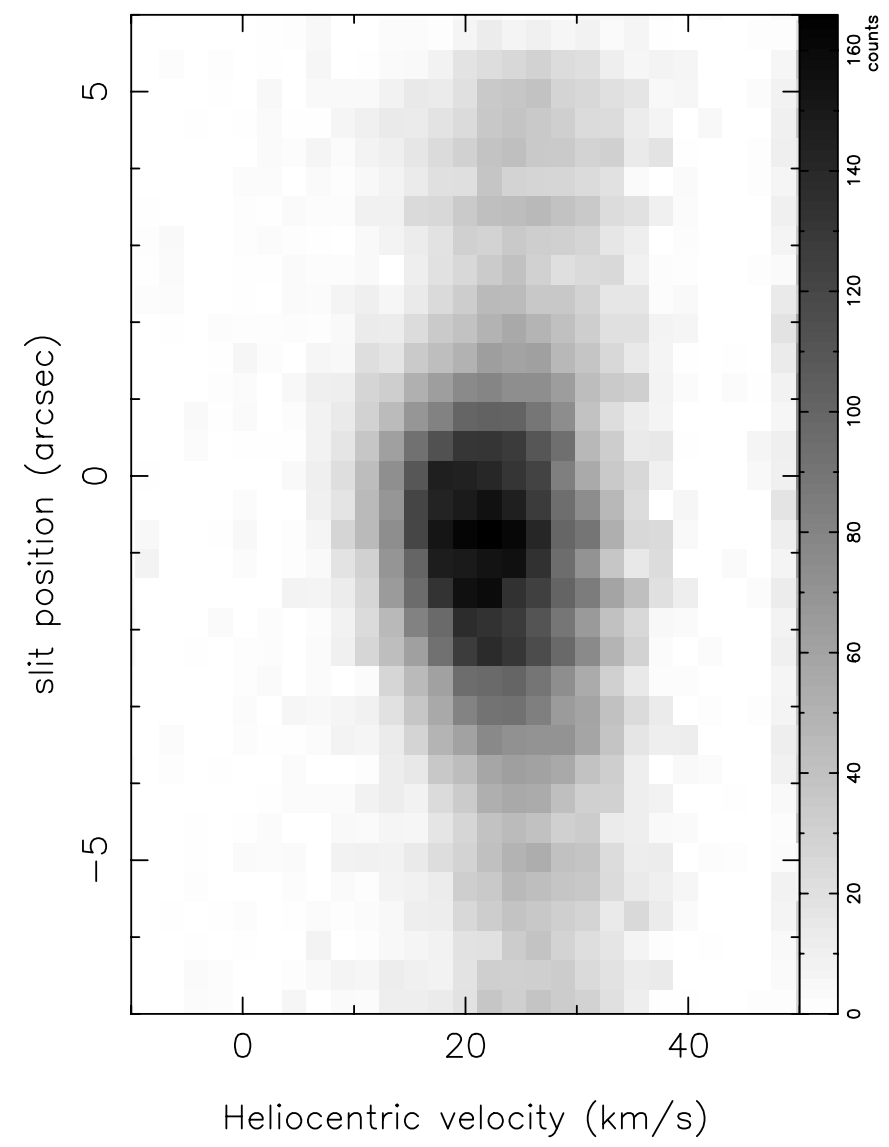

FIG. 3.-Position-velocity image of the extracted $\mathrm{D} \alpha$ line from the Keck spectrum of the giant proplyd $244-440$. The wing of the $H \alpha$ line has been fitted as a fourth-degree polynomial and removed to reveal the $\mathrm{D} \alpha$ profile. Velocities are heliocentric with respect to the rest wavelength of $\mathrm{D} \alpha$, and the intensity stretch is linear.

the samples from the different data sets are illustrated on a map of the Orion Nebula in Figure 1. All line velocities given are mean velocities, averaged over the line, weighted by the intensity. The given widths are 2.309 times the rms velocity width, which is equal to the FWHM if the profile is Gaussian, which is a good approximation. All widths are corrected for instrumental broadening.

The dominant sources of error in the extraction of the deuterium profiles are almost certainly systematic in nature, except for in a few instances (the higher lines of the CTIO spectra and in some regions of the KPNO spectra) where the photon $\mathrm{S} / \mathrm{N}$ is particularly low. We give uncertainties in the quantities in Table 1 that are subjectively estimated, based on the variations between the results of the three different subtraction techniques. These uncertainties do not include spatial variations in the measured quantities within a given region, which can be substantial in some cases. In the two cases where upper limits on $I(\mathrm{D}) / I(\mathrm{H})$ are given in the table, these are dominated by photon-counting noise, and the stated limits are $3 \sigma$. Note that HA do not explain the provenance of their quoted uncertainties and we feel that they may have substantially underestimated the importance of systematic errors (HB do not quote uncertainties in their values).

\subsection{KPNO Spectra}

The spectra obtained with the Kitt Peak $4 \mathrm{~m}$ echelle spectrograph have the widest spatial coverage of any of our 
TABLE 1

Characteristics of Deuterium Balmer LiNeS

\begin{tabular}{|c|c|c|c|c|c|c|}
\hline Position & Balmer Line & $\begin{array}{c}I(\mathrm{D}) / I(\mathrm{H}) \\
\left(\times 10^{4}\right)\end{array}$ & $\begin{array}{c}v(\mathrm{D}) \\
\left(\mathrm{km} \mathrm{s}^{-1}\right)\end{array}$ & $\begin{array}{c}\Delta v(\mathrm{D}) \\
\left(\mathrm{km} \mathrm{s}^{-1}\right)\end{array}$ & $\begin{array}{c}v(\mathrm{H}) \\
\left(\mathrm{km} \mathrm{s}^{-1}\right)\end{array}$ & $\begin{array}{c}\Delta v(\mathrm{H}) \\
\left(\mathrm{km} \mathrm{s}^{-1}\right)\end{array}$ \\
\hline \multicolumn{7}{|c|}{ Keck Spectra } \\
\hline \multirow{2}{*}{$177-341$ (nebula) ....... } & $\alpha$ & $3.5 \pm 1.0$ & $27.0 \pm 3.0$ & $17 \pm 3$ & $20.8 \pm 0.1$ & $25.8 \pm 0.1$ \\
\hline & $\beta$ & $6.0 \pm 2.0$ & & & $20.9 \pm 0.1$ & $25.3 \pm 0.1$ \\
\hline \multirow[t]{2}{*}{$170-337$ (nebula) ....... } & $\alpha$ & $3.3 \pm 1.0$ & $22.0 \pm 3.0$ & $16 \pm 3$ & $18.9 \pm 0.1$ & $23.2 \pm 0.1$ \\
\hline & $\beta$ & $6.0 \pm 2.0$ & & & $18.5 \pm 0.1$ & $23.2 \pm 0.1$ \\
\hline \multirow{2}{*}{$182-413$ (nebula) ....... } & $\alpha$ & $2.0 \pm 1.0$ & $25.0 \pm 3.0$ & $16 \pm 3$ & $14.2 \pm 0.1$ & $27.3 \pm 0.1$ \\
\hline & $\beta$ & $3.9 \pm 2.0$ & & & $14.2 \pm 0.1$ & $27.3 \pm 0.1$ \\
\hline \multirow[t]{2}{*}{$244-440$ (nebula) ....... } & $\alpha$ & $6.0 \pm 1.0$ & $24.5 \pm 1.0$ & $13 \pm 2$ & $14.5 \pm 0.1$ & $31.2 \pm 0.1$ \\
\hline & $\beta$ & $10.0 \pm 2.0$ & & & $14.5 \pm 0.1$ & $30.1 \pm 0.1$ \\
\hline \multirow[t]{2}{*}{$244-440$ (proplyd)...... } & $\alpha$ & $12.0 \pm 1.0$ & $21.5 \pm 1.0$ & $13 \pm 1$ & $14.5 \pm 0.1$ & $32.6 \pm 0.1$ \\
\hline & $\beta$ & $17.0 \pm 2.0$ & & & $14.0 \pm 0.1$ & $31.7 \pm 0.1$ \\
\hline \multicolumn{7}{|c|}{ KPNO Spectra } \\
\hline JW698 North ........... & $\alpha$ & $1.0 \pm 0.5$ & $25.0 \pm 3.0$ & $14 \pm 3$ & $14.4 \pm 0.5$ & $29.8 \pm 0.5$ \\
\hline JW698 Bar ............... & $\alpha$ & $7.5 \pm 1.0$ & $25.0 \pm 1.0$ & $12 \pm 2$ & $18.2 \pm 0.5$ & $28.9 \pm 0.5$ \\
\hline JW698 South ........... & $\alpha$ & $5.5 \pm 1.0$ & $24.0 \pm 3.0$ & $12 \pm 3$ & $13.5 \pm 0.5$ & $29.8 \pm 0.5$ \\
\hline JW352 North ........... & $\alpha$ & $4.0 \pm 1.0$ & $23.0 \pm 3.0$ & $13 \pm 2$ & $11.3 \pm 0.5$ & $27.1 \pm 0.5$ \\
\hline JW352 Center ........... & $\alpha$ & $<0.5$ & & $\ldots$ & $14.7 \pm 0.5$ & $36.2 \pm 0.5$ \\
\hline JW352 Bar ............. & $\alpha$ & $4.0 \pm 1.0$ & $25.0 \pm 2.0$ & $13 \pm 3$ & $20.0 \pm 0.5$ & $38.8 \pm 0.5$ \\
\hline \multicolumn{7}{|c|}{ CTIO Spectra } \\
\hline & $\alpha$ & $3.3 \pm 2.0$ & $29.1 \pm 1.0$ & $10 \pm 4$ & $16.1 \pm 0.2$ & $25.2 \pm 0.2$ \\
\hline & $\beta$ & $5.1 \pm 1.5$ & $30.5 \pm 0.5$ & $10 \pm 2$ & $16.2 \pm 0.2$ & $24.1 \pm 0.2$ \\
\hline & $\gamma$ & $9.3 \pm 1.6$ & $29.0 \pm 0.5$ & $11 \pm 1$ & $15.7 \pm 0.2$ & $24.1 \pm 0.2$ \\
\hline & $\delta$ & $14.3 \pm 2.8$ & $28.6 \pm 0.5$ & $12 \pm 1$ & $15.4 \pm 0.5$ & $23.6 \pm 0.5$ \\
\hline & $\epsilon$ & $15.7 \pm 7.1$ & $30.1 \pm 1.7$ & $13 \pm 4$ & $14.5 \pm 0.5$ & $24.2 \pm 0.5$ \\
\hline & $\zeta$ & $18.0 \pm 4.0$ & $27.6 \pm 1.0$ & $9 \pm 3$ & $13.4 \pm 1.0$ & $\ldots$ \\
\hline & $\eta$ & $12.0 \pm 4.0$ & $\ldots$ & $\ldots$ & $14.6 \pm 0.5$ & $24.6 \pm 0.5$ \\
\hline & $\theta$ & $<\overline{10} .2$ & $\ldots$ & $\ldots$ & $14.5 \pm 0.5$ & $24.2 \pm 0.5$ \\
\hline \multicolumn{7}{|c|}{ Canada-France-Hawaii Telescope Spectra (Hébrard et al. 2000a) } \\
\hline North ........... & $\beta$ & $4.2 \pm 1.1$ & $\ldots$ & $\ldots$ & $\ldots$ & $\ldots$ \\
\hline Center ................... & $\beta$ & $2.3 \pm 0.6$ & $\ldots$ & $\ldots$ & $\ldots$ & $\ldots$ \\
\hline \multirow[t]{2}{*}{ South ................... } & $\alpha$ & $2.2 \pm 0.2$ & $26.5 \pm 1.5$ & $8.6 \pm 1.0$ & $14.8 \pm 1.5$ & $32.0 \pm 0.5$ \\
\hline & $\beta$ & $5.7 \pm 1.1$ & $27.0 \pm 1.5$ & $8.6 \pm 1.0$ & $17.0 \pm 1.0$ & $32.1 \pm 0.5$ \\
\hline \multicolumn{7}{|c|}{ Very Large Telescope Spectra (Hébrard et al. 2000b) } \\
\hline & $\alpha$ & $\sim 2$ & $\ldots$ & $\sim 11$ & $\ldots$ & $\sim 30$ \\
\hline & $\beta$ & $\sim 6$ & $\ldots$ & $\sim 11$ & $\cdots$ & $\sim 30$ \\
\hline & $\gamma$ & $\sim 7$ & $\ldots$ & $\sim 11$ & $\ldots$ & $\sim 30$ \\
\hline & $\delta$ & $\sim 9$ & $\ldots$ & $\sim 11$ & $\ldots$ & $\sim 30$ \\
\hline & $\epsilon$ & $\sim 10$ & $\ldots$ & $\sim 11$ & $\ldots$ & $\sim 30$ \\
\hline
\end{tabular}

data sets but unfortunately only include the $\mathrm{H} \alpha$ line. The full extent of the two regions covered is shown by the dashed boxes in Figure 1. The original motivation for obtaining these spectra was the study of high-velocity features in the Orion nebula - the slits centered around JW698 include the bow shocks HH 203 and HH 204 at their southern end, while those centered around JW352 include the HH 202 region and various outflows from the embedded Orion-S region.

To improve the signal-to-noise ratio of the spectra, the three-dimensional position-velocity cube of each region was collapsed along one spatial dimension. In the case of the JW698 spectra, the bright bar is quite linear, at a position angle of $\simeq 50^{\circ}$, and this provides a natural spatial axis. Therefore, the position-velocity cube was collapsed along the direction parallel to the bar, effectively giving a synthetic slit oriented perpendicular to the bar. In the case of the JW352 spectra, the bar is more closely horizontal and is much less well defined, so we merely collapsed the spectra along the R.A. direction.

The presence of high-velocity HH flows in the regions studied complicates the analysis of the deuterium lines, since blueshifted $\mathrm{H}$ emission $v_{\text {hel }} \simeq-55 \mathrm{~km} \mathrm{~s}^{-1}$ can be mistaken for D emission at $v_{\text {hel }} \simeq 27 \mathrm{~km} \mathrm{~s}^{-1}$. However, the high-velocity emission is generally much broader than the deuterium lines, so spatial pixels that are contaminated by it can be easily identified by looking at the $\mathrm{H} \alpha$ intensity in the $v_{\text {hel }}=-75 \rightarrow-65 \mathrm{~km} \mathrm{~s}^{-1}$ band. Such pixels are rejected when collapsing the position-velocity cube, producing a synthetic slit spectrum that is largely free from high-velocity features. This technique works particularly well in the case of the emission near the bar in the JW698 spectra, as shown in Figure 4, since here the high-velocity emission (wing of HH 203) is oriented roughly perpendicular to the bar. The 

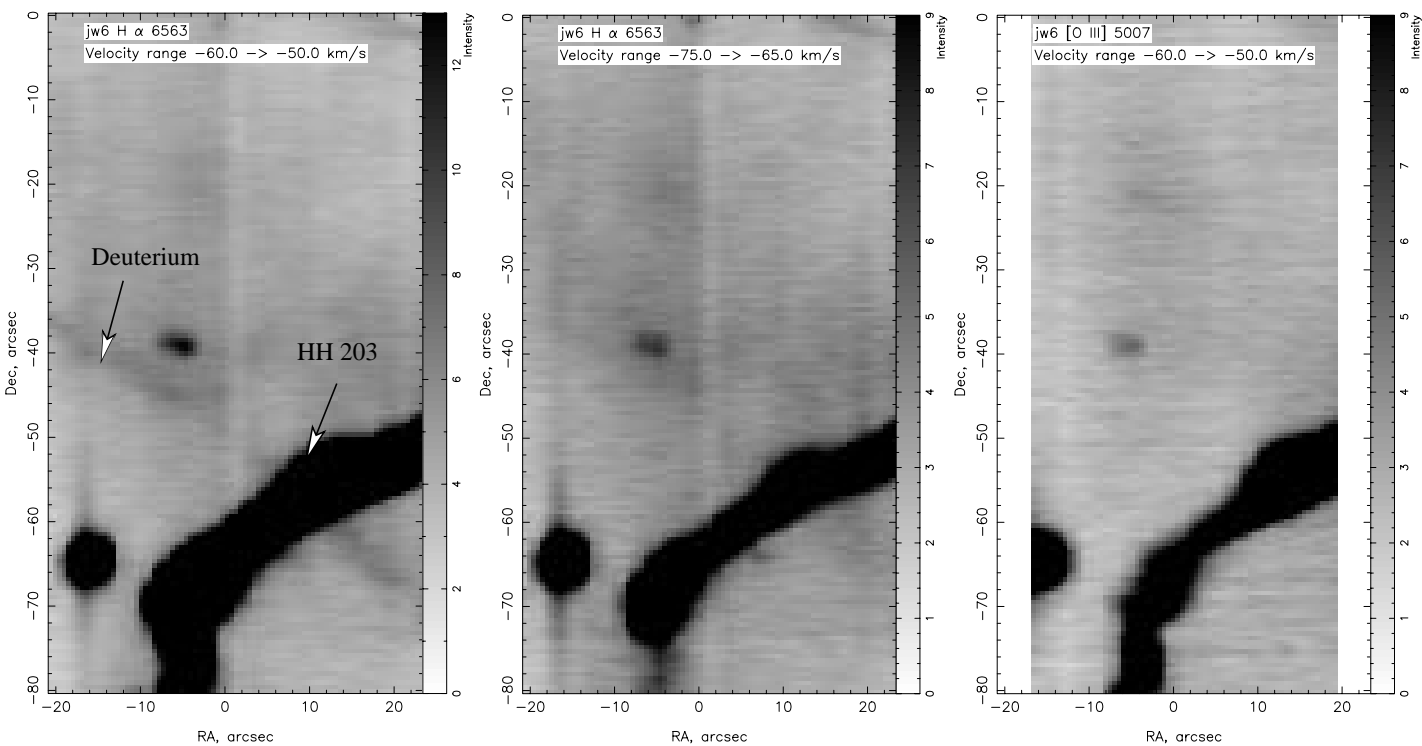

FIG. 4.-Isovelocity images of the region of the Orion bar near $\theta^{2}$ Ori A constructed from the KPNO long-slit spectra. The left panel shows blueshifted $\mathrm{H} \alpha\left(v_{\text {hel }}=-60 \rightarrow-50 \mathrm{~km} \mathrm{~s}^{-1}\right)$ plus systemic $\mathrm{D} \alpha\left(v_{\mathrm{hel}}=+14 \rightarrow+24 \mathrm{~km} \mathrm{~s}^{-1}\right)$. It is apparent that the deuterium emission from the bar $($ diagonal feature sloping from upper left to lower right) is clearly separated from the high-velocity hydrogen emission associated with HH 203 and HH 204 . The central panel shows hydrogen emission at velocities slightly blueward of the deuterium component, while the right panel shows [O III] $\lambda 4959$ emission in the same velocity range. The bar feature is completely absent in both these images.

technique was not successful in all cases. In particular, in the central and far-northern regions of the JW352 slits, the data were severely contaminated by high-velocity emission from HH 202 and other flows that proved impossible to remove.

In further areas of the spectra, there was no detectable deuterium component. In many cases, this can be ascribed to the general faintness of the emission from those zones (for example, the dark bay area to the north of the JW698 slits, where the foreground dust extinction is high). Such zones were not used in the analysis, apart from the region just above the bar in the JW352 slits (labeled "Center"), where the most stringent upper limit on $I(\mathrm{D}) / I(\mathrm{H})$ could be obtained. All regions covered by the spectra that were used in the deuterium analysis are shown by gray shading in Figure 1. These are divided into six areas (JW352 North, Center, and Bar and JW698 North, Bar, and South), in which the mean characteristics of the $\mathrm{D} \alpha$ and $\mathrm{H} \alpha$ lines are tabulated in Table 1. It is apparent that the intensity ratio $I(\mathrm{D}) / I(\mathrm{H})$ varies by at least an order of magnitude from place to place across the nebula. It is highest in the region of the bright bar and near $\theta^{2}$ Ori A (JW698 South) and lowest in the regions just northwest of the bar (JW698 North, JW352 Center), having an intermediate value nearer the Trapezium (JW352 North).

Figure 5 shows in more detail the spatial variation of the deuterium- and hydrogen-line intensities near the bar in the JW698 slits, together with those of other species of varying degrees of ionization. It is apparent from this figure that the high $I(\mathrm{D}) / I(\mathrm{H})$ in the bar is a geometric effect caused by the spatial separation of the hydrogen and deuterium emission layers. The main ionization front is seen tangentially at the position of the bar, allowing the ionization stratification to be directly seen. It is apparent from the figure that the deuterium emission peaks in a very thin layer $\left(\simeq 3^{\prime \prime}\right)$ close to the IF, slightly to the neutral side of the peak in the [S II] $\lambda 6731$ emission. The hydrogen emission, on the other hand, peaks in a broader $\left(\simeq 30^{\prime \prime}\right)$ recombination layer, displaced toward the ionizing stars of the Trapezium.

\subsection{Keck Spectra}

The data obtained with the Keck I HIRES spectrograph consist of short (14") slits, which were centered on four of the bright Orion proplyds $(170-334,177-341,182-413$, and 244-440). Although this data set is not as spatially extended as the KPNO one, the spectra have higher S/N and include a greater variety of emission lines, including $\mathrm{H} \beta$, and also have a slightly better velocity resolution. The highest $I(\mathrm{D}) / I(\mathrm{H})$ were found in the giant proplyd 244-440 and the adjacent nebula. This proplyd lies on the far side of

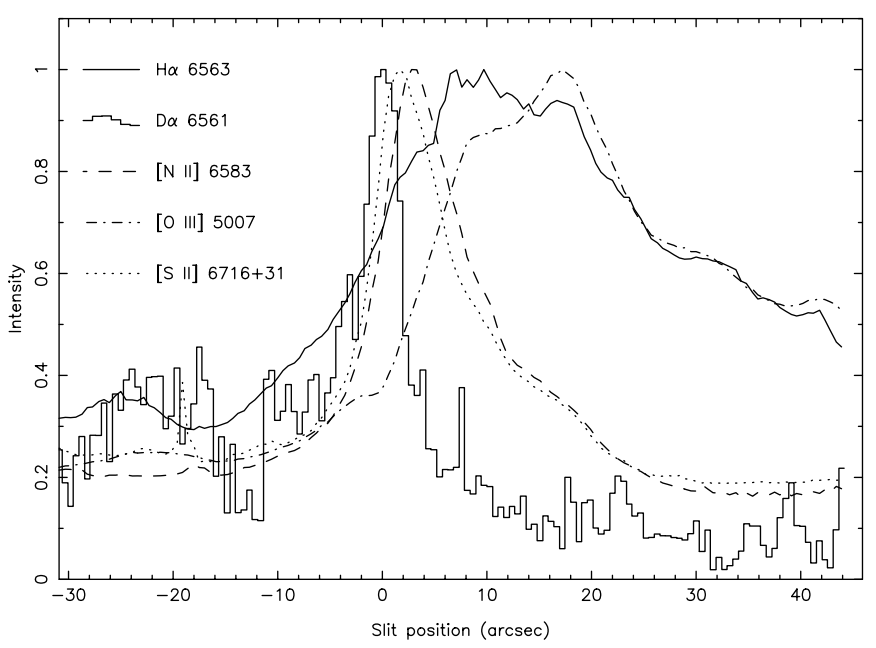

FIG. 5.-Spatial intensity profiles of various emission lines along a line perpendicular to the bright bar. The spatial scale increases toward the northwest, with the Trapezium stars lying off the graph at a position of $\simeq 120^{\prime \prime}$. 


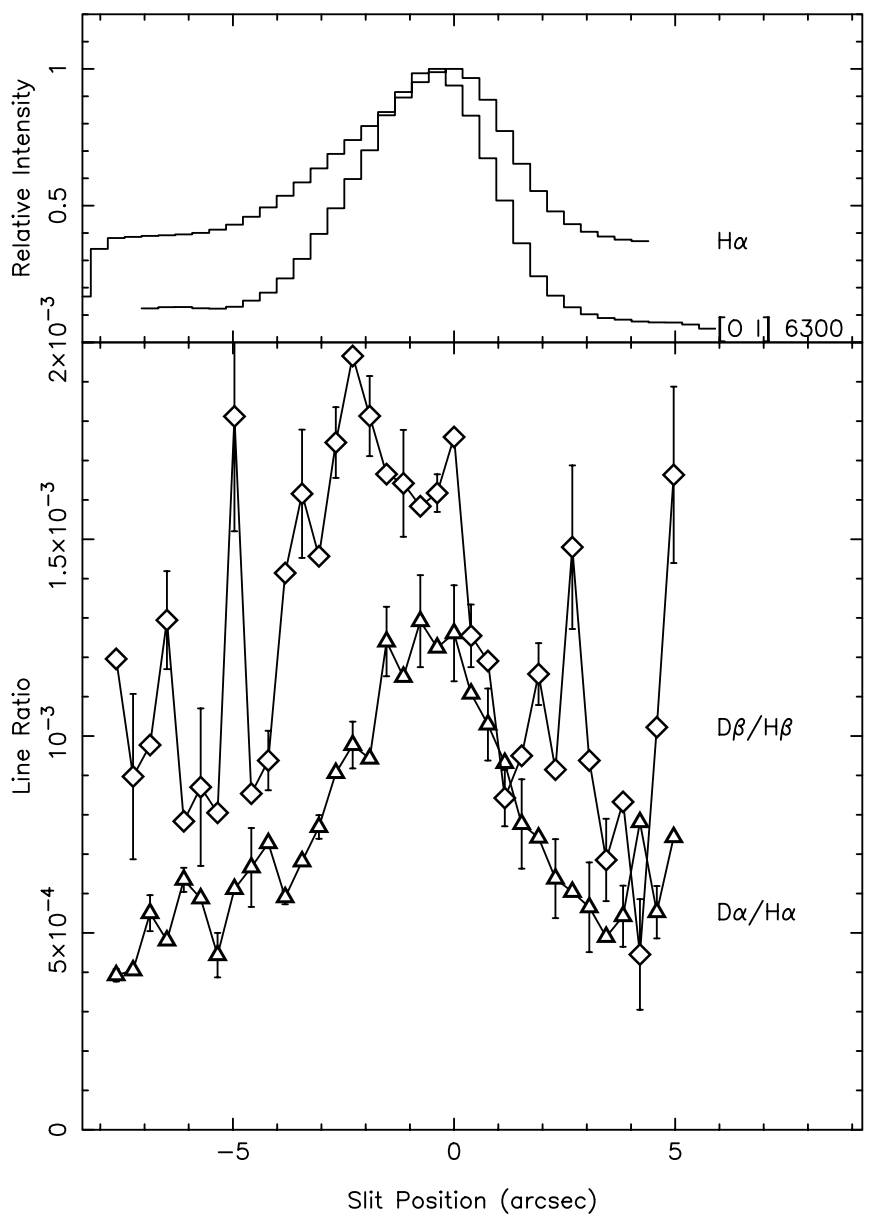

FIG. 6.-Line intensities as functions of position along the slit, for the giant proplyd $244-440$, with the spatial scale arcseconds relative to the peak of the proplyd $\mathrm{H} \alpha$ emission. The upper panel shows the relative intensities of $\mathrm{H} \alpha$ and [O I $] \lambda$ 26300 . The proplyd can be seen to fill about two-thirds of the slit and is slightly brighter in $\mathrm{H} \alpha$ than the background nebula at its peak. The lower panel shows the spatial variations in the $I(\mathrm{D}) / I(\mathrm{H})$ ratios along the slit for $\mathrm{H} \alpha$ and $\mathrm{H} \beta$. Error bars on this and subsequent plots are $\pm 1 \sigma$ and represent the variations found when using different orders of polynomial $(n=3-5)$ to subtract the underlying wing of the $\mathrm{H}$ line. For all the graphs, note that the horizontal alignment of the curves for different emission lines may be in error by 1-2 pixels.

the bar from the Trapezium, close to $\theta^{2}$ Ori A. Figures 6, 7, and 8 show the measured line characteristics as a function of position along the slit.

It can be seen from Figure 6 that $I(\mathrm{D}) / I(\mathrm{H})$ is significantly larger for $\mathrm{H} \beta$ than for $\mathrm{H} \alpha$ and shows a marked increase for both lines at the position of the proplyd. Figures 7 and 8 show that the deuterium lines are extremely similar to the [O I] $\lambda 6300$ line in their kinematics, further evidence that the deuterium lines arise very close to the ionization front.

The proplyds $170-334$ and $177-341$ both show extensive blueshifted high-velocity emission, so it was possible to measure the deuterium component only from the background nebula in these cases. The same was true for $182-413$ because of the very low contrast of this proplyd against the background. Deuterium-line characteristics for all these positions are given in Table 1 . As in the case of $244-440$, it can be seen that $I(\mathrm{D}) / I(\mathrm{H})$ is always higher for $\mathrm{H} \beta$ than for $\mathrm{H} \alpha$, although the absolute level is about 2 times smaller in these cases.

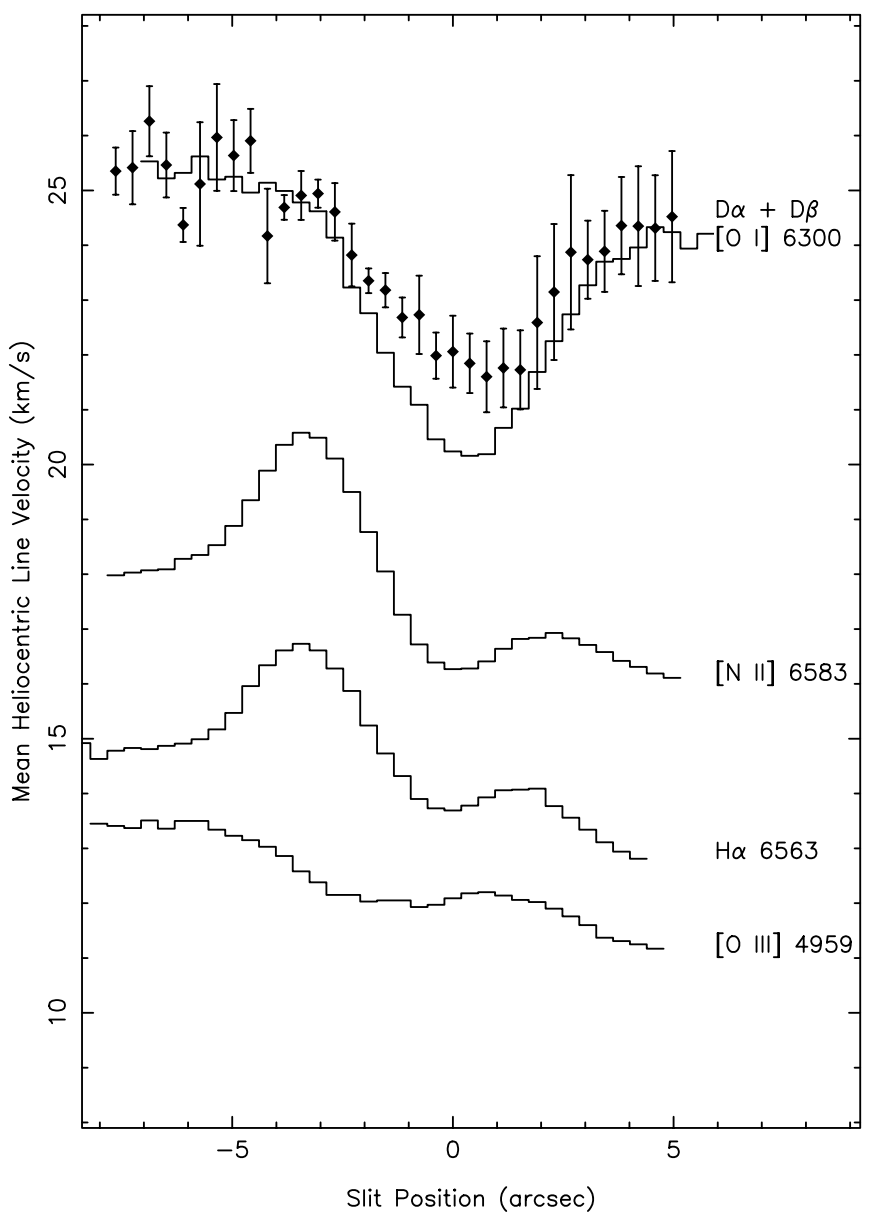

FIG. 7.- Same as Fig. 5, but showing mean heliocentric velocity of each emission line. The results for $\mathrm{D} \alpha$ and $\mathrm{D} \beta$ have been averaged and are shown by filled symbols.

\subsection{CTIO Spectra}

These data come from observations described in Baldwin et al. (2000). Although they cover only a small spatial region (near the brightest part of the nebula; see Fig. 1), they have a much greater spectral range, covering the entire Balmer series, and hence usefully complement our other data sets. Characteristics of the measured deuterium components of these lines are given in Table 1 . It can be seen that the $I(\mathrm{D}) / I(\mathrm{H})$ ratio increases as one ascends the series, at least as far as $\mathrm{H} \delta(6-2)$. There is some evidence for a turnover in this trend in the higher members of the series, but the data for these lines are very noisy. There is no evidence for any significant variations in the peak velocity or width of different lines of the series.

\subsection{Consistency between the Different Data Sets}

Inspection of Table 1 shows no obvious inconsistencies between the results obtained from the different data sets presented in this paper. However, there are some differences between our results and those of HA and HB. In particular, in our Keck and KPNO spectra, we find significantly larger line widths for the D component (13-16 $\mathrm{km} \mathrm{s}^{-1}$ ) than those reported by $\mathrm{HA}\left(\simeq 9 \mathrm{~km} \mathrm{~s}^{-1}\right)$. This may in part be due to the different methods employed (HB report larger widths, of $\sim 11 \mathrm{~km} \mathrm{~s}^{-1}$ for the same position as HA). On the other 


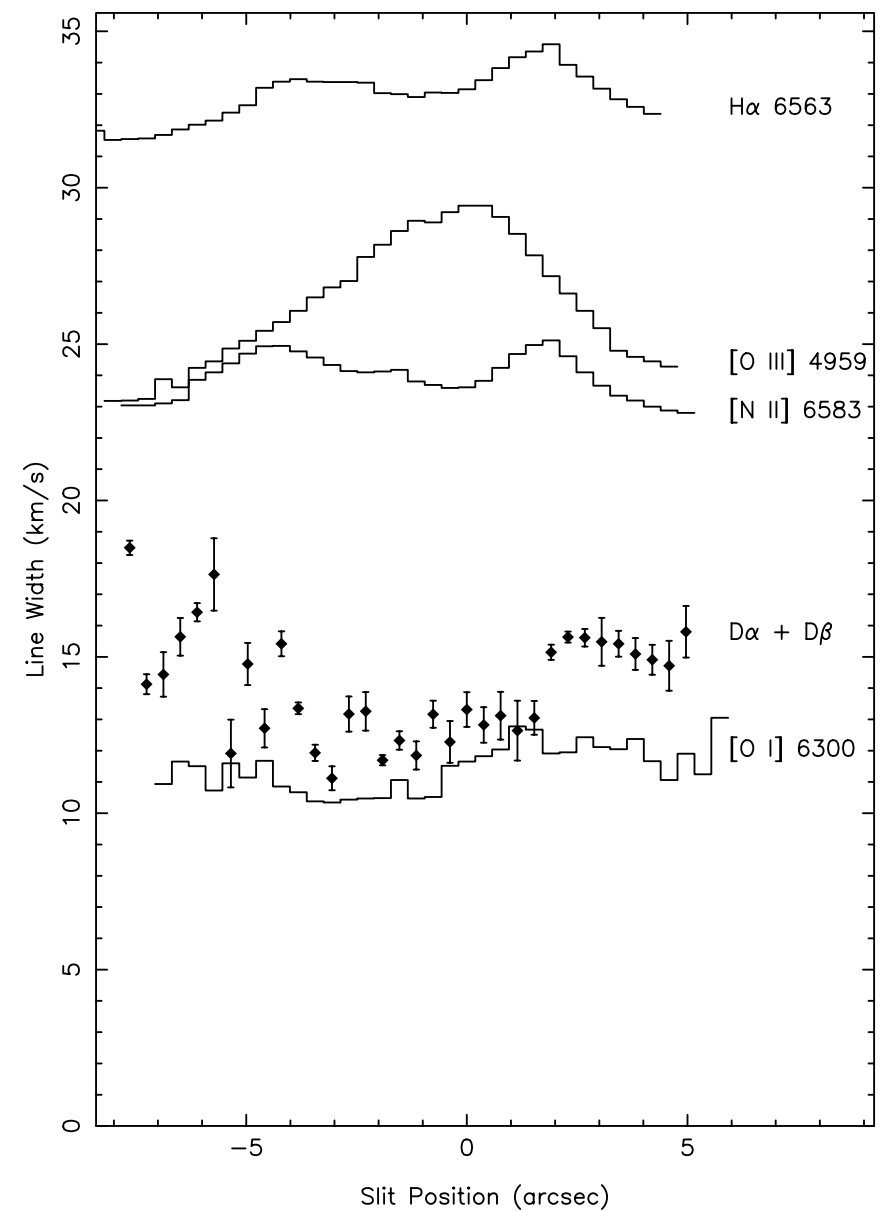

FIG. 8.-Same as Fig. 6, except showing rms velocity width of each emission line, scaled to be equal to the FWHM in the case of a Gaussian profile. The results for $\mathrm{D} \alpha$ and $\mathrm{D} \beta$ are presented in the same manner as in Fig. 6. The widths are corrected for instrumental broadening $\left(\mathrm{FWHM}=6.2 \mathrm{~km} \mathrm{~s}^{-1}\right)$.

hand, there may be real variations across the face of the nebula, as evidenced by our CTIO spectra, which also show smaller line widths (see Table 1).

The Balmer decrements reported by HB are also somewhat flatter than we measure from the CTIO spectra (Table 1). However, the difference is probably not significant, given our estimated uncertainties and the different extraction techniques used.

\section{PREDICTED DEUTERIUM-LINE INTENSITIES}

As indicated in HA, the observed D lines are the result of continuum fluorescence by radiation from $\theta^{1}$ Ori $\mathrm{C}$ at the deuterium Lyman lines. In this section we will present estimates of the expected relative intensities of the $\mathrm{D}$ and $\mathrm{H}$ lines, compare these with the observations, and comment on how the theoretical model can be improved. We present a considerably more refined predictive model than that of HA in their discovery paper.

\subsection{The Region Where the $\mathrm{D}$ Lines are Formed}

Because the D emission occurs near or within the PDR, a good model for this region is necessary, which we computed using Version 95.00 of the spectral synthesis code CLOUDY (Ferland 2001). This was done for the region $37 \mathrm{~W}$ (BFM) and continues into the PDR until $\mathrm{CO}$ and $\mathrm{H}_{2}$ have fully formed. A constant gas pressure was assumed.
We also assumed a plane-parallel geometry for the illuminating radiation, even though we know that the $\mathrm{H}$-line emission occurs closer to $\theta^{1}$ Ori $\mathrm{C}$ than the fluorescent $\mathrm{D}$ emission. This effect is not large since the scale height of the recombination-line emission is only $0.025 \mathrm{pc}$ (Pogge, Owen, $\&$ Atwood 1992; O'Dell 2001), while the distance from $\theta^{1}$ Ori $\mathrm{C}$ to the ionization front is about $0.25 \mathrm{pc}$ (O'Dell 2001). The small scale height for the emission is derived from the fact that the emission layer thickness is $0.1 \mathrm{pc}$ if the assumption is made that it is at a constant density. If it has an exponential distribution, then the density varies with a scale height of $0.05 \mathrm{pc}$ and the emissivity drops with a scale height of $0.025 \mathrm{pc}$, which is consistent with the CLOUDY model shown in Figure 9.

Continuum pumping rates and line self-shielding are treated as in Ferland (1992) in the calculations we present below. The results can be simplified with the rule that line pumping mostly occurs through the Doppler core, occurs
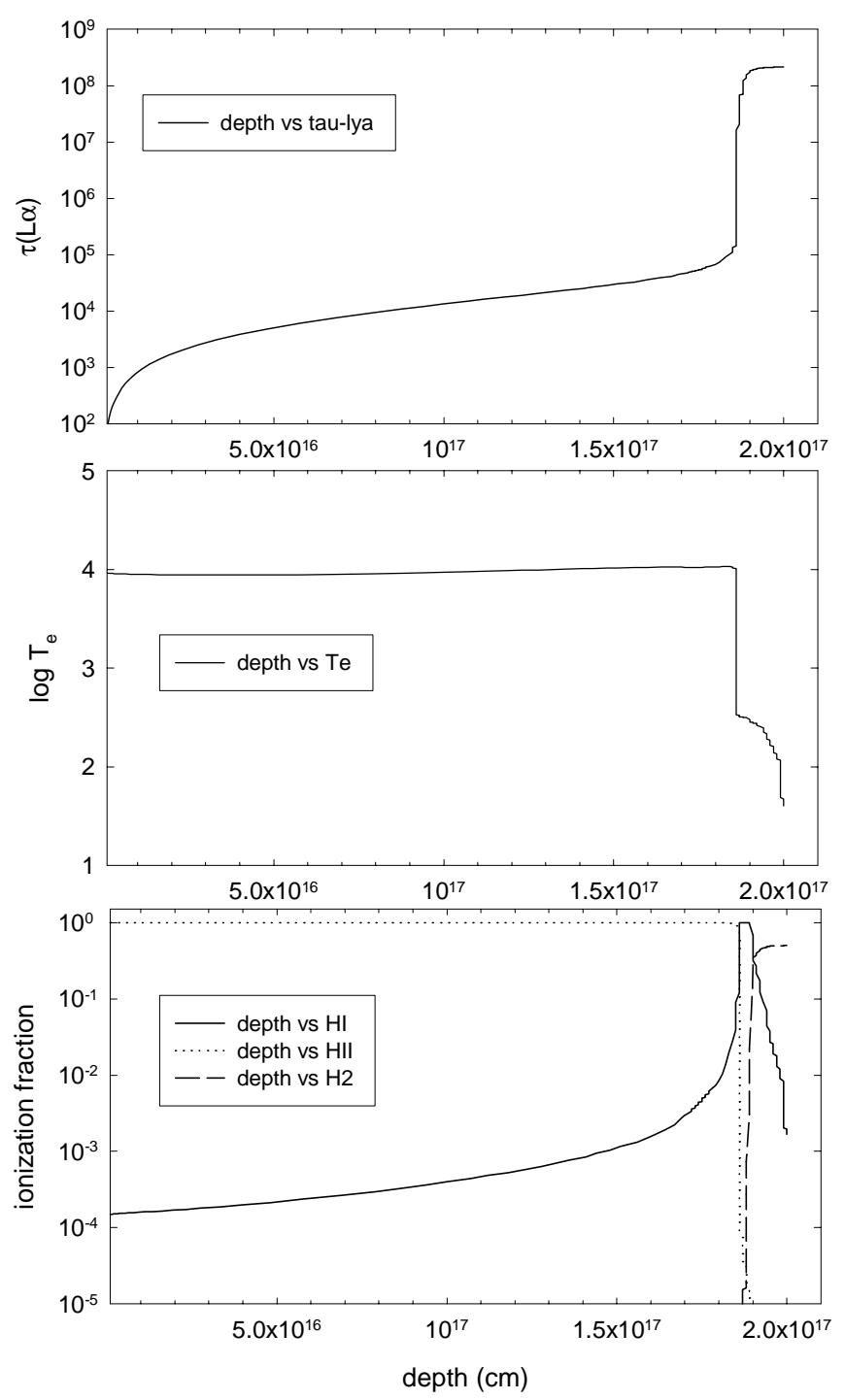

FIG. 9.- Ionization, thermal, and optical depth structure of the Orion $\mathrm{H}$ II region and PDR as calculated by CLOUDY using the parameters of BFM as described in the text. The independent axis is the depth into the layer measured from the illuminated face of the cloud. From top to bottom, the panels show the optical depth in Ly $\alpha$, the electron temperature, and the hydrogen atomic, ionic, and molecular fractions $(\mathrm{H}$ is fully $\mathrm{H}_{2}$ at the shielded face). 
around optical depth unity, and will remove typically 1 Doppler width of the continuum.

Figure 9 shows some of the details of the computed structure. The independent axis is the depth into the layer, measured from the illuminated face of the cloud. The lower panel shows the hydrogen ionization structure, the hydrogen ionization front occurring at a depth of about $2 \times 10^{17}$ $\mathrm{cm}$. The middle panel shows the computed electron temperature, which is about $10,000 \mathrm{~K}$ in the $\mathrm{H}$ II region and falls to PDR temperatures of about $100 \mathrm{~K}$ across a large region and eventually to around $20 \mathrm{~K}$, where the gas is fully molecular $\left(\mathrm{H}_{2}\right.$ and $\left.\mathrm{CO}\right)$. The top panel shows the line-center optical depth of $\mathrm{Ly} \alpha$ for a layer assumed to be static and with a microturbulence of $10 \mathrm{~km} \mathrm{~s}^{-1}$. The local line width is self-consistently computed from the local kinetic temperature and this microturbulence. The figure shows that Ly $\alpha$ is quite optically thick throughout the entire plotted region. However, the $L y \alpha$ optical depth is only a point of reference, since the observed $\mathrm{D}$ lines are produced by excitations to $n \geq 3$. We will present our results in terms of the properties of $\operatorname{Ly} \beta$, even though it only contributes to one of our reference lines, D H $\alpha$. The other levels of $\mathrm{D}$ will be pumped by the higher Lyman-line photons in proportion to their optical depths and scattering properties as discussed later in this section. Each level will contribute to the emission from the lower members of the D Lyman series.

$\operatorname{Ly} \beta$ has a line-center opacity about one-fourth that of Ly $\alpha$, and $\mathrm{D}$ has an abundance about $1.5 \times 10^{-5}$ that of $\mathrm{H}$ (Linsky 1998), so that the point where fluorescent production of $\mathrm{H} \alpha$ is the most efficient $\left[\tau(\operatorname{Ly} \alpha) \simeq 4 \times 10^{5}\right]$ occurs not in the PDR itself but right at the $\mathrm{H}$ I-H II interface, a conclusion insensitive to the $\mathrm{D} / \mathrm{H}$ abundance ratio over a large range of values.

The location of where the deuterium emission is produced may be reflected in the width of the lines. Our line widths, corrected for instrumental broadening, average to be $12.8 \pm 2.2 \mathrm{~km} \mathrm{~s}^{-1}$, which is larger than the value of $8.6 \pm 1.0 \mathrm{~km} \mathrm{~s}^{-1}$ found in HA. Although the narrowness of the lines confirms that the lines are being formed in a region of lower temperature, their width may have a nonthermal component that arises from acceleration of material within the ionization front.

\subsection{The Balmer $\mathrm{D} n / \mathrm{H} n$ Spectrum}

\subsubsection{Overview}

While the deuterium lines are formed by fluorescence with the far-UV continuum at and slightly beyond the ionization front, the hydrogen lines are emitted during recombination of photoionized gas in the $\mathrm{H}$ in region. The Orion Nebula is a blister of ionized gas on the observer's side of the Orion molecular cloud so that it is optically thick in Lyman continuum radiation, which means that the surface brightness in a hydrogen recombination line will be proportional to the incident flux of ionizing photons (BFM) while the rate of fluorescent excitation of the deuterium atoms will be proportional to incident flux of nearultraviolet photons. Since both of these fluxes scale inversely with the square of the distance to $\theta^{1}$ Ori $\mathrm{C}$, the ratio of the $\mathrm{D} / \mathrm{H}$ Balmer lines will depend primarily upon the temperature of the dominant star. This means that cooler photoionizing stars will in general have higher $\mathrm{D} / \mathrm{H}$ Balmer line ratios, since there will be relatively more lower energy, nonphotoionizing radiation. It is this reason why the D lines were discovered by HA at a relative intensity much more than anticipated from the expected abundance and pure recombination.

\subsubsection{The Hydrogenic Model Atom}

The adopted model for hydrogen was one in which individual $l$ states within each $n$ state were considered separately; i.e., it does not consider mixing between the $l$ states due to collisions. This assumption is justified at the densities encountered in Orion, at least for the low- $n$ states that are the primary contributors to the observed lower members of the Balmer sequence. We also calculated for reference the fully mixed (collision dominated) model and found quite different results for the efficiency of producing the lines and in the resultant deuterium Balmer decrements.

The driving continuum $\eta(\mathrm{Ly} n)$ excites the $n p$ level of deuterium. Subsequent cascades are treated by finding the sum

$$
\sum_{l=2}^{l<n} A_{n l}
$$

of all transition probabilities down from an upper level $n$ and then assuming that the fraction of excitations that follow a particular route $n l \rightarrow n^{\prime} l^{\prime}$ is $A_{n l}$ divided by this sum. Transition probabilities for each $n l \rightarrow n^{\prime} l^{\prime}$ transition were obtained from the radial integral of the hydrogenic wave function. The term in the summation is essentially Seaton's cascade matrix, although it was more expedient to compute the sums on the fly rather than using tabulated values of the matrix.

\subsubsection{The Pump-Cascade Efficiency}

It is convenient to define the pumping efficiency as the number of deuterium $\mathrm{H} \beta$ photons produced for each photon absorbed by deuterium $\operatorname{Ly} \beta, N(\mathrm{D} \beta) / N(\operatorname{Ly} \beta)$. Since absorption of the higher lines scales from this line, it does not matter that absorptions of $\operatorname{Ly} \beta$ photons do not actually produce an $\mathrm{H} \beta$ photon. The pumping efficiency depends on two parts, the flux distribution of the continuum from 912 to $1216 \AA$ and the ratio of the continuum near $\operatorname{Ly} \beta$ to the total number of ionizing photons from the star $[Q(\mathrm{H})]$.

The $\mathrm{D}$ atom is pumped by the series of Lyman-line photons. Each Lyman line Lyn can absorb a stellar continuum flux $f_{\lambda}$. The number of photons that the atom absorbs per unit time and area, $\eta(\operatorname{Ly} n)$, is the number of photons that occurs over a velocity line width $\delta V$ and is given by

$$
\eta(\mathrm{Ly} n)=\frac{\delta V}{c} \frac{\lambda f_{\lambda}}{h v} \mathrm{~cm}^{-2} \mathrm{~s}^{-1}
$$

The Lyman-line opacities do not directly enter as long as the lines are optically thick and absorb the continuum across their Doppler core. The situation that we envision is most closely approximated by "case C," the case where the atom is fluoresced through the Lyman-line photons (Ferland 1999).

The pumping efficiency will then become

$$
\frac{N(\mathrm{D} \beta)}{N(\mathrm{Ly} \beta)}=\frac{f(\mathrm{H} \beta)}{h v} \frac{c}{\delta V} \frac{h v}{\lambda f_{\lambda}}
$$

\subsubsection{The Case A-Case B Distinction}

Having established the excitation rate, we must now determine the cascades that produce the observed lines. 
There are two limiting cases. Case A applies when Lymanline photons either freely escape the nebula or are destroyed. Case B applies when all Lyman lines scatter often enough to be converted into a Balmer line plus Ly $\alpha$ or two-photon emission, requiring that typical Lyman-line photons scatter a dozen or more times. This is an excellent description of the hydrogen problem but will not necessarily apply to deuterium in this environment.

A simple calculation shows that case A may be more appropriate for D I. Consider a typical Lyman line, the $1 s-4 p$ transition. This can directly lead to $\mathrm{D} \beta$ if the decay is to $n=3$. For reference, the transition probabilities for lower levels are $A(4 p-1 s)=6.81 \times 10^{7} \mathrm{~s}^{-1}, A(10 p-1 s)=$ $4.21 \times 10^{6} \mathrm{~s}^{-1}$, and $A(20 p-1 \mathrm{~s})=5.23 \times 10^{5} \mathrm{~s}^{-1}$, and the branching ratio for producing a $4 p-1 s$ transition from $n=4$ is 0.839 , so that half a dozen scatterings are enough to degrade the transition into case $B$.

If the Lyman line escapes or is destroyed before these scatterings occur, then case $\mathrm{A}$ is the best approximation. The continuum pumping occurs at line-center optical depth unity, and re-emitted photons have half a chance of being re-emitted in the hemisphere toward the star and so escape. The other half are emitted in the opposite direction and see a substantial optical depth. Many of these photons will still manage to escape from the illuminated face since they stand a $50 \%$ chance of re-emission into the open hemisphere on each scattering.

What is the fate of those Lyman-line photons that repeatedly scatter deeper into the cloud? The dominant opacity source at these wavelengths will be grains, with a typical cross section per atom of $6 \times 10^{-22} \mathrm{~cm}^{-2}$ proton ${ }^{-1}$ around $1000 \AA$ (BFM). The $4 p-1 s, 10 p-1 s$, and $20 p-1 s$ transitions have line-center cross sections of about $2.6 \times 10^{-15}$, $1.6 \times 10^{-16}$, and $2.0 \times 10^{-17} \mathrm{~cm}^{-2}$ proton $^{-1}$, for the assumed velocity field. If the $\mathrm{D} / \mathrm{H}$ abundance ratio is $1.5 \times 10^{-5}$, then the absorption cross sections per nucleon are, for this set of transitions, $3.9 \times 10^{-20}, 2.4 \times 10^{-21}$, and $3.0 \times 10^{-22} \mathrm{~cm}^{-2}$. Lyman-line photons above $n=10$ will probably be absorbed by grains before they scatter often enough to be degraded into Balmer-line photons. Lower lines are probably not destroyed by grains but still have a substantial chance of escaping the nebula.

The conclusion of this section is that the atom is not likely to be well into either the case A or case B limits, but is likely to be closer to case A. We will present results for both cases below, but will consider case $\mathrm{A}$ as the standard model.

\subsubsection{Hydrogen Lines Blocking the Incident Continuum}

There are two ways through which hydrogen affects the fluorescent excitation of deuterium, the first being damping in the wings of the strongest hydrogen Lyn lines and the second being the pseudocontinuum caused by the overlapping of the higher Lyn lines.

The damping wings of hydrogen lines compete with deuterium in absorbing photons. The D lines are $~ 3.7$ Doppler widths away from the $\mathrm{H}$-line center, placing them in the damping wings of the transition. The damping constants for the $\mathrm{Ly} \alpha, \mathrm{Ly} \beta$, and $\mathrm{Ly} \gamma$ transitions are $\sim 3.61 \times 10^{-4}$, $4.82 \times 10^{-5}$, and $1.38 \times 10^{-5}$, so the H Lyn opacities are factors of $a / x^{2} \simeq 2.5 \times 10^{-5}, 3.5 \times 10^{-6}$, and $0.96 \times 10^{-6}$ smaller at the position of the D Lyn line. For a D/H abundance ratio of $1.5 \times 10^{-5}$ (Linksy 1998), the $\mathrm{D}$ Ly $\alpha$ line will be well shielded by the $\mathrm{H} \operatorname{Ly} \alpha$ line, the D Ly $\beta$ line marginally so, while the D Ly $\gamma$ line will see the full continuum.
Clearly, this will be very sensitive to the precise $\mathrm{D} / \mathrm{H}$ ratio. In the following we will consider both the case where the Ly $\beta$ line sees the full continuum and no continuum at all. This will mainly affect the Balmer $\alpha / \beta$ intensity ratio. If one or the other case can be ruled out by observations, then something might be said about the $\mathrm{D} / \mathrm{H}$ abundance ratio.

At higher members of the Lyman series, the deuterium lines begin to fall within the core of the adjacent hydrogen lines, thereby drastically reducing the radiation field. For this reason we assume that only the lowest 20 Lyman lines see the incident stellar continuum.

\subsubsection{Grains Blocking the Incident Continuum}

Imbedded grains can also suppress the stellar continuum, thus affecting the rate of fluorescent pumping of deuterium. Figure 10 shows the optical depth in grains of the continuum as a function of the optical depth in the core of the deuterium $\operatorname{Ly} \beta$ line as calculated for BFM's region $37 \mathrm{~W}$ with CLOUDY. This assumes that the grains have an absorption cross section of $6 \times 10^{-22} \mathrm{~cm}^{2}$ proton $^{-1}$ around $1000 \AA$ and that there is no strong wavelength dependence over the $912-1216 \AA$ region. One sees that the grain continuum optical depth $\left(\tau_{\text {grain }}\right)$ is about unity at an optical depth of 1 for the deuterium $\operatorname{Ly} \beta$ line (which is where the pumping is most efficient). Fortunately, this result is relatively insensitive to the assumed $\mathrm{D} / \mathrm{H}$ abundance ratio, as most of the increase of the deuterium $\operatorname{Ly} \beta$ optical depth occurs as transition is made past the ionization front. In our calculations we have adopted $\tau_{\text {grain }} \simeq 1$. There will be an additional effect of the grains in that the optical deuterium lines will suffer greater extinction than the corresponding hydrogen line $(\sim 0.1 \mathrm{mag})$ because of the fact that the deuterium emission arises from farther into the nebula.

\subsubsection{The Form of the Driving Continuum}

To complete the calculation, we needed values of the stellar continuum $f_{\lambda}$ at the wavelengths of all the deuterium Ly $n$ lines. These $f_{\lambda}$ could be found from continua predicted from stellar atmosphere calculations, but this introduced a dependence on the details of these calculations.

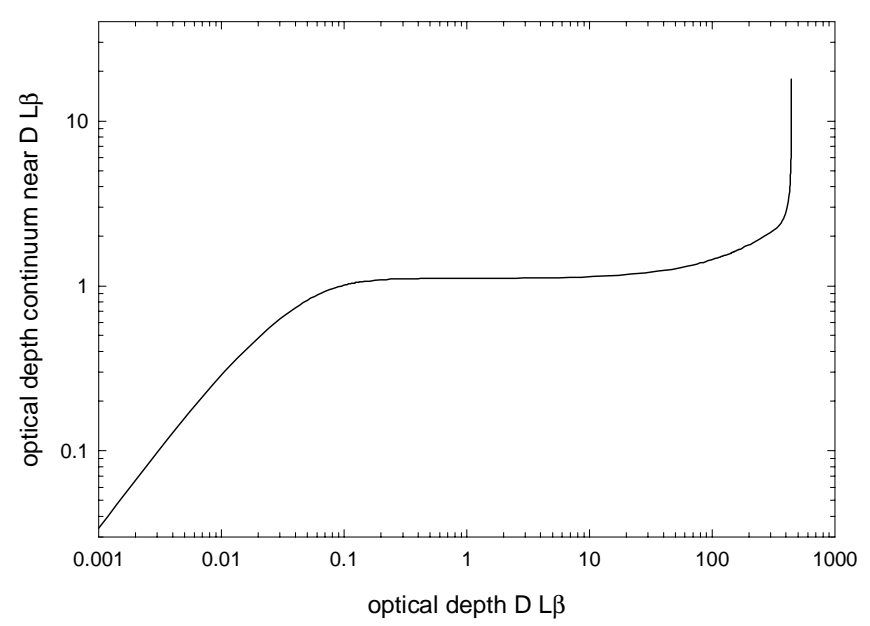

Fig. 10.-Dependence of the optical depth in grains (at Ly $\beta$ ) is shown as a function of the optical depth in the center of the deuterium $\operatorname{Ly} \beta$ line, as calculated for BFM's region $37 \mathrm{~W}$. Although a $\mathrm{D} / \mathrm{H}$ abundance ratio of $1.5 \times 10^{-5}$ was assumed, the grain optical depth at the point where the line optical depth reaches unity is relatively insensitive to the assumed abundance. 
We considered stellar continua from the CoStar (Schaerer \& de Koter 1997) and Kurucz (1991) grids to test dependencies, but mainly relied on the Phoenix atmospheres, produced by Peter Hauschildt and collaborators, since these include full non-LTE and winds. Jason Aufdenberg kindly provided access to the O-star grid produced as part of his Ph.D. thesis. This is at four temperatures and with both static and windy atmospheres, all having $\log g=3.9$.

Aufdenburg also provided predictions of the emergent $f_{\lambda}$ in the wings of the Lyman lines. The deuterium lines are shifted by $-82 \mathrm{~km} \mathrm{~s}^{-1}$ relative to the hydrogen lines by the isotope effect. The motion of $\theta^{1}$ Ori $C$ relative to the PDR is unknown since the radial velocity of the star is poorly known. We assumed that the star moves by $10 \mathrm{~km} \mathrm{~s}^{-1}$ relative to the PDR, so the stellar continuum $70 \mathrm{~km} \mathrm{~s}^{-1}$ blueward of the line center was taken as the driving continuum. Figure 11 shows the predicted $f_{\lambda}$ for each deuterium Lyn line relative to $f_{\lambda}$ at the deuterium $\operatorname{Ly} \beta$ line. There is considerable scatter, but these suggest that $f_{\lambda} \simeq$ constant is roughly appropriate, as would be expected for farultraviolet wavelengths for a stellar temperature near $40,000 \mathrm{~K}$.

\subsubsection{Efficiency of the Standard Model}

We define our "standard model" as one with case A Lyman lines with the continuum blocked by Lyman lines with $n \leq 3$, no grain absorption, a collision-free $n l$-resolved atom, with irradiation by an $f_{\lambda} \simeq$ constant continuum. The continuum is suppressed by Lyman-line core absorption for $n>20$. Clearly, there are uncertainties associated with these assumptions, and their effects will be estimated as we present the results.

The results of computing the fluorescence efficiency for the standard model is that $N(\mathrm{D} \beta) / N(\operatorname{Ly} \beta)=0.272$. If pumping to the $n=54$ level had been included, this would have increased the efficiency value to 0.637 . If case B had been assumed, these values would increase to 1.66 and 3.93 .

\subsubsection{The Predicted $\mathrm{D} / \mathrm{H}$ Line Ratios}

Having established the number of cascade photons per driving photon, we could now predict the $\mathrm{D} \beta / \mathrm{H} \beta$ intensity

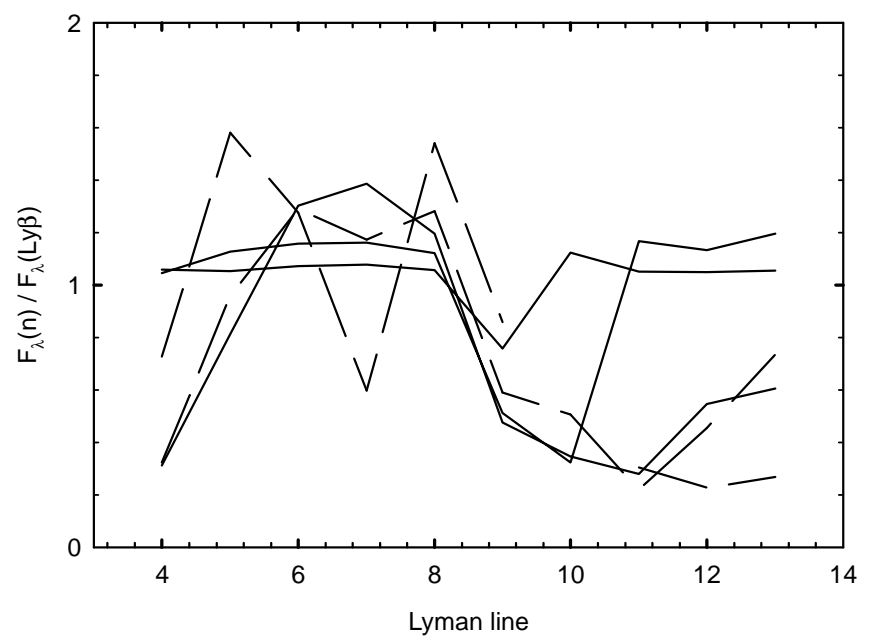

FIG. 11.-Flux in the continuum $70 \mathrm{~km} \mathrm{~s}^{-1}$ blueward of line center for the lowest 13 Lyman lines are shown, all relative to the flux in the continuum blueward of $\operatorname{Ly} \beta$. The solid lines are for the static and dashed for the windy Phoenix atmospheres. ratio. The total number of $\mathrm{D} \beta$ photons will be

$$
N(\mathrm{D} \beta)=\frac{n(\mathrm{D} \beta)}{n(\mathrm{Ly} \beta)} \frac{\delta V}{c} \frac{\lambda f_{\lambda}}{h v},
$$

while the number of hydrogen $\mathrm{H} \beta$ recombination photons will be

$$
N(\mathrm{H} \beta)=\frac{\alpha_{\mathrm{H} \beta}^{\mathrm{eff}}(T)}{\alpha_{\mathrm{B}}(T)} Q(\mathrm{H}),
$$

both in units of $\mathrm{s}^{-1}$.

The ratio of the recombination coefficients (the $\alpha$ terms in eq. [4]) is 0.12 for these conditions. Although case B probably does not apply to the deuterium lines, it certainly does describe the hydrogen lines. The ratio of the pumped $\mathrm{D} \beta$ to the recombination $\mathrm{H} \beta$ is then

$$
\begin{aligned}
\frac{N(\mathrm{D} \beta)}{N(\mathrm{H} \beta)} & =\frac{\eta(\mathrm{D} \beta)}{\eta(\mathrm{Ly} \beta)} \frac{\alpha_{\mathrm{B}} T}{\alpha_{\mathrm{H} \beta}^{\text {eff }}(T)} \frac{\delta V}{c} \frac{\lambda f_{\lambda} / h v}{Q(\mathrm{H})} \\
& \simeq 8.55 \times 10^{6} \delta V_{22} \frac{\lambda f_{\lambda}}{Q(\mathrm{H})} \eta_{2},
\end{aligned}
$$

where $\delta V_{22}$ is the intrinsic FWHM line width in units of 22 $\mathrm{km} \mathrm{s}^{-1}$ and $\eta_{2}$ is the cascade efficiency in units of 0.27 .

Equation (5) does not include the correction for grain extinction in the $\lambda \sim 1000 \AA$ continuum. Accordingly, the $\mathrm{D} / \mathrm{H}$ line ratio predicted by equation (5) has been reduced by a factor of 0.37 to account for this.

Figure 12 shows the $\mathrm{D} \beta / \mathrm{H} \beta$ line ratio for various stellar models over a range of effective temperatures. The intensity ratio decreases with increasing stellar temperature since hotter stars emit proportionately more ionizing radiation relative to the continuum around $\operatorname{Ly} \beta$. The observed ratio in the Trapezium region is (4-6) $\times 10^{-4}$.

\subsubsection{Comparison of Predicted and Observed $\mathrm{D} \beta / \mathrm{H} \beta$ Line Ratios}

Baldwin et al. (2000) compare their observed emissionline ratios with values predicted by CLOUDY using these and similar stellar atmosphere models. From this they reach certain conclusions about the effective stellar temperature, which can be used here. They conclude that the stellar temperature is close to $36,000 \mathrm{~K}$ and that the Kurucz and CoStar atmospheres are not as applicable as some others.

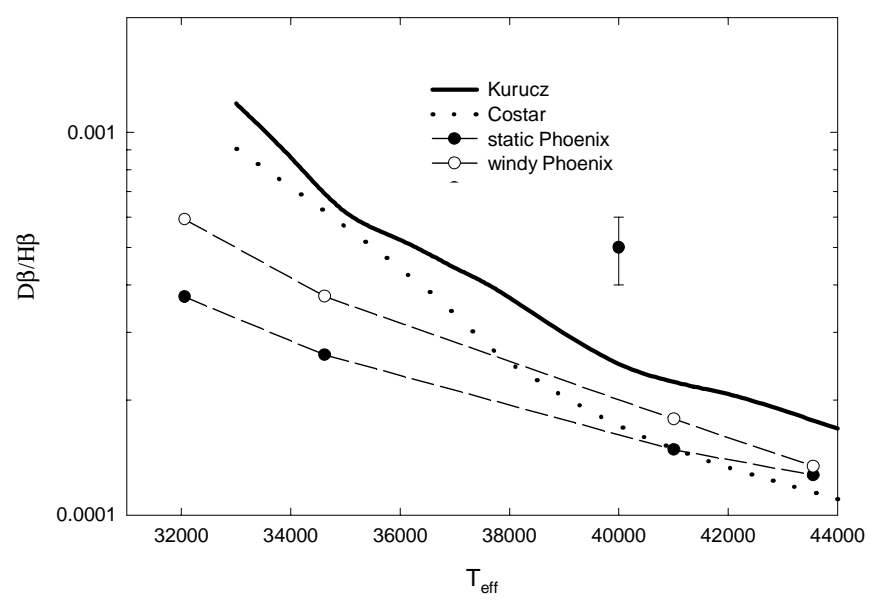

FIG. 12.-Ratio of the fluorescent excited $\mathrm{D} \beta$ to the recombination $\mathrm{H} \beta$ line is shown for various stellar effective temperatures and models. The ratio predicted by eq. (5) has been reduced by a factor of 0.37 to account for UV grain extinction. The observed ratio is $(4-6) \times 10^{-4}$ and is indicated by the filled circle with error bars. 
This means that our preferred models are the two Phoenix predictions. Using a temperature of $36,000 \mathrm{~K}$ and the middle Phoenix values leads to a predicted $\mathrm{D} \beta / \mathrm{H} \beta$ line ratio of $3 \times 10^{-4}$, similar but slightly less than the observed range within the nebula, i.e., excluding the much larger value at the giant proplyd 244-440.

Our model has considered only the radiation from $\theta^{1}$ Ori $\mathrm{C}$ as a source of the ionizing radiation that drives the hydrogen emission and the near ultraviolet radiation that causes the deuterium fluorescence. If one were to add the effects of the other Trapezium stars, which are cooler and less luminous, then the theoretically predicted values would come into better agreement with the observations.

The agreement of the predictions and the observations may be fortuitous owing to the simplicity of the model. There may be partial conversion to case B for the lower Lyman lines, which would increase the line ratio, thus closing the gap with the observations, but could easily exceed the observed ratio if a sufficient approximation of case B obtains.

Varying and partial conversion of Lyman lines into case B, l-mixing, and grain extinction of the UV all may explain why the $\mathrm{D}$ I/H I line ratios vary as much as they do. Continuum pumping alone results in a $\mathrm{D} \beta / \mathrm{H} \beta$ ratio that is set only by the continuum shape and should always be the same for any gas that is excited by $\theta^{1}$ Ori C.

\subsubsection{The Deuterium Balmer Decrement}

In addition to the line ratios at $\mathrm{H} \beta$, variations in the line ratios for various members of the Balmer series can also be a useful diagnostic. This was first indicated in the studies of $\mathrm{HA}$ and $\mathrm{HB}$, and our larger database allows this to be extended. In principle, what one wants to do is to compare the Balmer decrements of the $\mathrm{H}$ I and D I lines. In practice, this is best done by looking at variations in the line ratios (D I/H I) for the various members of the Balmer series. There are several particular advantages of this approach, including the facts that it does not involve comparing weak extracted lines with one another and it removes the effects of variable interstellar extinction (with both wavelength and position).

Figure 13 gives the normalized (to Balmer $\beta$ ) line ratios for all of the samples in Table 1. Since there is no evidence for spatial variation in the decrements, we have combined data from different positions. The $\mathrm{H} \alpha$ point comes from the Keck spectra of $244-440$, while the other points come from the CTIO spectra. We have lumped together the line ratios for upper levels 6-9 and have estimated the upper limit for H10. Examination of Figure 13 indicates that for the lowest transitions there is good agreement with the standard model in the variations of the line ratios for various low values of $n$. However, for the higher $n$ values a systematic difference appears. The most likely explanation of this is that one is seeing the effect of the higher hydrogen Lyman lines shielding the UV continuum. Our model assumes that this sets in abruptly at $n=21$, whereas the transition may occur at a lower value of $n$.

\subsubsection{The Bry Lines}

The good velocity agreement of the weak-line component blueward of $\mathrm{Br} \gamma$ in the spectrum of Oudmaijer et al. (1997) strongly argues for its being the same deuterium emission that we see in the Balmer lines. It is difficult to assess the relative strength of the two lines from the plot presented in their paper, but we estimate it to be $\mathrm{D}(\mathrm{Br} \gamma)$ /

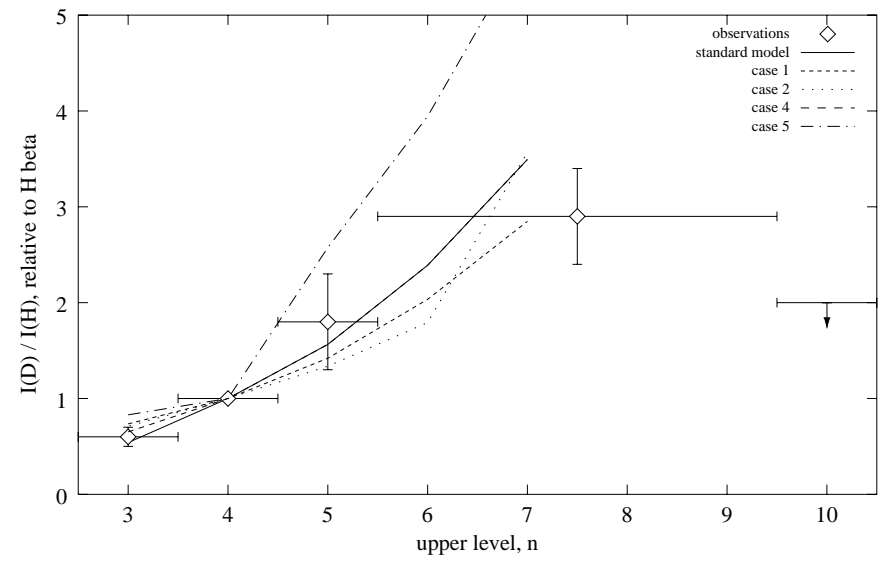

FIG. 13.-Deuterium-to-hydrogen line ratios, normalized to the value at $\mathrm{H} \beta$, are shown as a function of the upper level $n$ value. The results for levels 6-9 have been averaged together, and the value at $n=10$ is an estimated upper limit. The solid line represents the predicted values for the standard model. As discussed in the text, the deviations at the highest $n$ values are probably due to shielding of the UV continuum by the highest hydrogen Lyman lines.

$\mathrm{H}(\mathrm{Br} \gamma)=6 \times 10^{-3}$. Using our measured ratio of $\mathrm{D}(\mathrm{H} \epsilon) /$ $\mathrm{H}(\mathrm{H \epsilon})$ of $1.57 \times 10^{-3}$ gives for the ratio of the two deuterium lines with the same upper level $\mathrm{D}(\mathrm{Br} \gamma) / \mathrm{D}(\mathrm{H} \epsilon) \simeq 0.65$, whereas the theoretical ratio for $T=10^{4}$ is 0.17 ; i.e., the $\mathrm{D}(\mathrm{Br} \gamma)$ line is some 4 times too large. Since this value is derived from multiplying uncertain numbers and it is difficult to know the uncertainty of the $\mathrm{D}(\mathrm{Br} \gamma)$ line, the agreement has to be considered as acceptable.

\subsubsection{Detectability of Deuterium Recombination Lines}

As well as the fluorescent component to each deuterium Balmer line, there should also be a recombination component, which forms in the ionized gas in an identical way to the hydrogen Balmer lines, with the exception that the deuterium recombination lines should be closer to case A since the $\mathrm{H}$ II region will be marginally optically thin to the deuterium Lyman lines. For example, the intensity ratio $I(\mathrm{D} \alpha) / I(\mathrm{H} \alpha)$ for this component should be $\simeq 0.67$ times the $\mathrm{D} / \mathrm{H}$ abundance ratio, with similar results for other lines of the series (the recombination Balmer decrements vary very little between case A and case B). Since the physics involved is so much simpler than in the case of the fluorescent component, there is the real prospect of determining the deuterium abundance if the recombination component can be detected. Figure 2 shows as dashed lines a $250 \times$ magnification of the expected deuterium recombination component for various abundances up to $10^{-4}$, compared with the same magnification of our best-quality observed line profile. It can be seen that, even at the highest abundance, the recombination component would probably not be detectable in our data. We estimate an upper limit to the $\mathrm{D} / \mathrm{H}$ abundance by this method to be $2 \times 10^{-4}$, which is roughly an order of magnitude larger than the expected value. It seems unlikely that improved future observations will be able to detect this component since such a detection would require a very large $\mathrm{S} / \mathrm{N}$ and a good understanding of the behavior of the wings of both the $\mathrm{H} \alpha$ line and the $\mathrm{D} \alpha$ fluorescent component.

\section{SUMMARY}

We have presented the results of analysis of multiple spectra of the Orion Nebula, demonstrating that the dis- 
covery observations of HA and HB are basically correct. These observations extend to further along the Balmer series than had previously been done. We derive predicted intensities and Balmer decrements for the deuterium lines from detailed models of the hydrogen and deuterium atoms and the assumptions that the deuterium lines are produced near the ionization front at the edge of the PDR and that the hydrogen lines arise from photoionization and recombination in the main emitting layer of the nebula. When one considers the complexity of the model and its numerous simplifying assumptions, excellent agreement is found between the observations and the predictions.

A regrettable outcome of the good basic agreement of the predictions of our model with the observations is that it is unlikely that one can use similar observations for determination of the $\mathrm{D} / \mathrm{H}$ abundances. This is because the processes that produce the deuterium and hydrogen emission are basically photon-counting mechanisms, so that objectto-object variations will primarily be due to the temperature of the dominant illuminating star. There is a strong observational selection effect operating in Galactic H II regions such that the optically bright objects are selectively those with blister geometries similar to Orion's; hence, the same abundance-insensitive processes will be operating in most objects.

We are grateful to Jason Aufdenberg for sharing his Arizona State stellar atmosphere model calculations. John Stauffer of the Center for Astrophysics graciously shared his Keck I observing time, which lead to the HIRES observations used in this study. Takao Doi of Rice University shared his KPNO $\mathrm{H} \alpha$ observations made for mapping the high-velocity components of Orion, and Jack Baldwin of Michigan State gave us digital copies of his CTIO spectra. C. R. O.'s financial support for this work was derived in part from STScI grant GO-8121 and the Alexander von Humboldt Foundation of Germany. G. J. F.'s nebular astrophysics research is supported by the NSF through AST 00-71180 and NASA through NAG5-8212. W. J. H. acknowledges financial support from DGAPA-UNAM project IN117799 and from CONACyT project E-25470, México.

\section{REFERENCES}

Baldwin, J. A., Ferland, G. J., Martin, P. G., Corbin, M. R., Cota, S. A., Peterson, B. M., \& Slettebak, A. 1991, ApJ, 374, 580 (BFM)

Baldwin, J. A., Verner, E. M., Verner, D. A., Ferland, G. J., Martin, P. G., Korista, K. T., \& Rubin, R. H. 2000, ApJS, 129, 229

Bertoldi, F., Timmermann, R., Rosenthal, D., Drapatz, S., \& Wright, C. M. 1999, A\&A, 346, 267

Ferland, G. J. 1992, ApJ, 389, L63

. 1999, PASP, 111, 1524

. 2001, PASP, 113, 41

Hébrard, G., Péquignot, D., Vidal-Madjar, A., Walsh, J. R., \& Ferlet, R. 2000a, A\&A, 354, L79 (HÄ)

Hébrard, G., Péquignot, D., Walsh, J. R., Vidal-Madjar, A., \& Ferlet, R. 2000b, A\&A, 364, L31 (HB)
Henney, W. J., \& O’Dell, C. R. 1999, AJ, 118, 2350

Kurucz, R. L. 1991, in Precision Photometry: Astrophysics of the Galaxy, ed. A. C. D. Philip, A. R. Upgren, K. Janes, \& B. Strömgren (Schenectady: Davis), 27

Linsky, J. L. 1998, Space Sci. Rev., 84, 285

O'Dell, C. R. 2001, PASP, 113, 29

Pogge, R. W., Owen, J. M., \& Atwood, B. 1992, ApJ, 399, 147

Oudmaijer, R. D., Drew, J. E., Barlow, M. J., Crawford, I. A., \& Proga, D. 1997, MNRAS, 291, 110

Schaerer, D., \& de Koter, A. 1997, A\&A, 322, 598

Traub, W. A., Carleton, N. P., \& Hegyi, D. J. 1974, ApJ, 190, L81

Vogt, S. S., et al. 1994, Proc. SPIE, 2198, 362 